\title{
Temporal summation at the warmth threshold*
}

\author{
JOSEPH C. STEVENS, WILLIAM C. OKULICZ, and LAWRENCE E. MARKS \\ John B. Pierce Foundation Laboratory and Yale University, New Haven, Connecticut 06519
}

\begin{abstract}
Threshold levels for warmth aroused by infrared irradiation were measured in six Ss at durations between 0.05 and $10 \mathrm{sec}$. Beyond a critical duration of about a second, the threshold does not depend on duration. Below critical duration time $t$ trades for irradiance $\varphi$ by the formula $\varphi=k t^{-\mathbf{0 . 8 2}}$. That these properties do not depend much on areal extent of stimulation was demonstrated by a study that compared temporal summation for two different areas of the same S's skin. Individual differences in apparent absolute sensitivity were explored under the rubric of the theory of signal detection.
\end{abstract}

Temporal summation is a property of nearly every sense modality, but its parameters have been more thoroughly mapped in some modalities than in others. The warmth sense is one that has received inadequate attention. Figure 1 brings together the results of all the studies on the subject that we could find. ${ }^{1}$ They come from three laboratories but were obtained from a total of only four Ss. Two of the experiments used infrared irradiation as the stimulus, and the third used microwave irradiation. Only threshold measurements were made; there is nothing on summation at supraliminal levels of warmth.

The three studies agree that summation takes place over brief durations, and in each case the results plot as straight lines in log-log coordinates. Beyond this, the picture is one of inconclusion and apparent disagreement. For one thing, the slopes of the lines vary considerably. Microwaves yielded a slope of -0.7 (Eijkman \& Vendrik, 1961). Infrared waves yielded a slope of -1.1 in one study (Hendler, Hardy, \& Murgatroyd, 1963) and about -0.5 in another (Geblewicz, 1935). The dotted line in Fig. 1 (slope equals -0.5) depicts Geblewicz's hypothesis that irradiance trades with the square root of duration to preserve threshold. The positions of the two functions for infrared waves are also very different.

Two of the functions also fail to give any estimate of the critical duration-the duration beyond which summation no longer takes place. Eijkman and Vendrik's study suggests a critical duration for microwaves in the vicinity of $2.5 \mathrm{sec}$.

To help improve our knowledge of temporal summation, the present study examined a larger number of Ss over a wider range of durations. The measurements reported here are confined to threshold, but work in progress aims to extend the measurement to the whole dynamic range of the warmth sense.

\section{METHOD}

\section{Apparatus}

The heat source was a quartz lamp (General Electric T-3)

*This research was supported by United States Public Health Service Grant No. ES00354-05. mounted in a parabolic cylindrical reflector. Its radiant output was controlled with a voltage regulator and calibrated with a Hardy radiometer (Hardy, Wolff, \& Goodell, 1952). Duration of stimulation was controlled with an electrical timer and a fast solenoid-driven shutter (opening and closing time negligible compared with exposure durations). The $\mathrm{S}$ rested his forehead against a cork-lined aluminum shield having a rectangular aperture of $21.8 \mathrm{~cm}^{2}$ or $5.72 \mathrm{~cm}^{2}$. Over this area, the density of radiant flux varied less than $7 \%$. To insure uniform absorption of the spectrum emitted by the lamp, the forehead was blackened with India ink (Hardy, Wolff, \& Goodell, 1952). Air temperature was held constant at $21^{\circ} \mathrm{C}$, relative humidity at $30 \%$.

\section{Procedure}

In the main experiment, thresholds were measured in six male Ss at each of 12 durations between 0.05 and $10 \mathrm{sec}$. Area of irradiation was $21.8 \mathrm{~cm}^{2}$. On the basis of pilot experiments, the

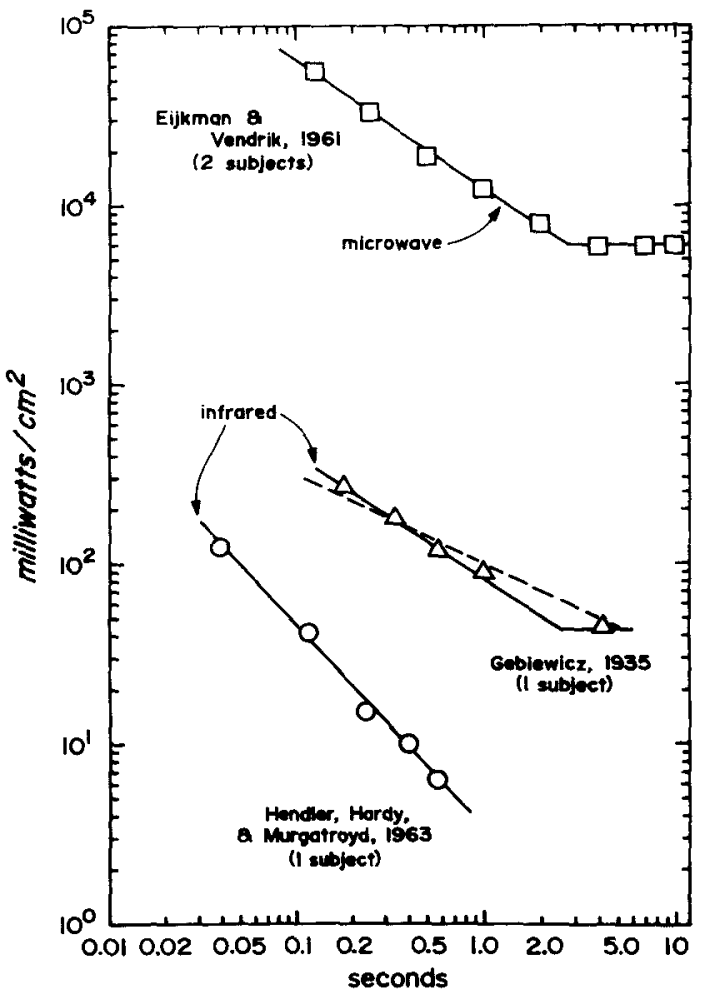

Fig. 1. Temporal summation at the warmth threshold, showing how stimulus irradiance depended on duration in three earlier studies. 


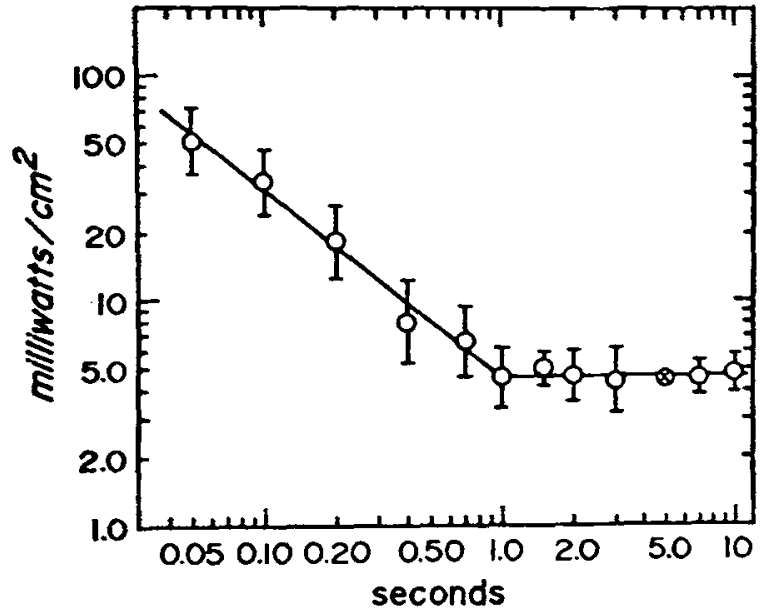

Fig. 2. Temporal summation at the warmth threshold. The size of the stimulus was $21.8 \mathrm{~cm}^{2}$. Plotted are the geometric averages for six Ss. The vertical lines represent plus and minus one standard deviation of the normalized log thresholds.

method of limits (ascending series only) was chosen. This method helps to circumvent problems of adaptation and permits the determination of a threshold in reasonable time. The threshold was reckoned as the geometric average of 12 successive determinations of the level at which the $S$ reversed from negative to positive judgment. In any run, the voltage was increased by $2.5-\mathrm{V}$ steps (roughly $5 \mathrm{~mW} / \mathrm{cm}^{2}$ ). Pilot work showed that intervals of about $10 \mathrm{sec}$ between stimulations were sufficient to avoid serious problems of adaptation.

The Ss were asked to arrive for the experiment in approximately "neutral" thermal state. A session then began with $15 \mathrm{~min}$ adaptation to the conditions of the experimental room. After some practice, a threshold was measured for a given duration, followed by a short rest period before changing to another duration. The order of durations was irregular and varied from $S$ to $S$. A session lasted 2 or $3 \mathrm{~h}$.

A secondary experiment explored the possibility that areal extent of stimulation might play a part in temporal summation. In the same test session, thresholds were measured for areas of $5.72 \mathrm{~cm}^{2}$ and $21.8 \mathrm{~cm}^{2}$, each at 11 different durations between 0.05 and $7.5 \mathrm{sec}$. Combinations of area and duration were tested in random order. It was, incidentally, impossible for the $S$ to distinguish which of the two areas was being stimulated. The $S$ (L.H.) took part in five complete test sessions spaced out over a period of 3 months.

Because of large individual differences among the $S s$ in the main experiment, the method of confidence rating was used in another secondary study of two $S s$, under the rubric of the theory of signal detection. The thresholds of these two Ss differed by nearly tenfold when measured by the method of limits. The question is whether or not individual criteria alone explain so large a difference.

In this experiment, on a given trial the $\mathrm{S}$ had to decide whether a stimulus was presented (signal) or withheld (no signal) and then to rate the certainty of his judgment on a scale from 1 to 3 , in order of increasing certainty (see Green \& Swets, 1966). A session began with several practice trials ("previews"), in which the $S$ was told whether or not the signal was present. Thereafter, a session comprised four blocks of 100 trials, separated by $10-\mathrm{min}$ rest periods. Within each block, there were 50 presentations of the signal and 50 "blanks," interspersed at random. To prevent response to extraneous cues, the $S$ was blindfolded and the shutter mechanism sounded exactly as on signal trials. The $S$ received no feedback on individual trials, but at the end of the session was paid $5 \mathrm{c}$ for every correct response and fined $5 \mathrm{c}$ for every error. [Although trial-by-trial feedback is a common procedure in signal detection experiments, it has been shown (Gundy, 1961) that feedback has little or no influence on the results, except at the beginning of a test session; even this initial effect is negligible if the $S$ is given some "preview" trials.]

S T.L. served in two sessions using a constant stimulus intensity of $2.5 \mathrm{~mW} / \mathrm{cm}^{2}$ and in two sessions using $1.0 \mathrm{~mW} / \mathrm{cm}^{2}$. S D.R. served in two sessions using $7.5 \mathrm{~mW} / \mathrm{cm}^{2}$ and two sessions using $2.5 \mathrm{~mW} / \mathrm{cm}^{2}$. Stimulus duration was always $3 \mathrm{sec}$, and areal extent $21.8 \mathrm{~cm}^{2}$.

\section{RESULTS AND DISCUSSION}

\section{Trading Functions and Critical Duration}

In Fig. 2, the threshold level (geometric average of the six Ss' results) is plotted as a function of duration in $\log -\log$ coordinates. Two straight lines have been fitted to the data: one horizontal segment beyond $1 \mathrm{sec}$ and another below $1 \mathrm{sec}$ having a slope equal to -0.82 . Like several other sense modalities, there appears to be a maximum or "critical" duration beyond which there is no further temporal integration and, in terms of the level of irradiance, the threshold is constant. In other words, if a person fails to perceive a radiant stimulation within a second or so after onset, he will not perceive it at any duration up to at least $10 \mathrm{sec}$. This is a remarkable feature of the warmth sense, because the skin temperature rises continuously over the whole period of stimulation. This point will receive considerable attention below.

Over durations shorter than $1 \mathrm{sec}$, it is possible to trade time $t$ for irradiance $\varphi$ to preserve the absolute threshold. The rule of trading approximates a general hyperbola having the equation

$$
\varphi=\mathrm{kt}^{-0.82} \text {. }
$$

In other words, a given percentage change in intensity is slightly more effective than the same percentage change in duration. The general hyperbola has often been used to describe temporal and spatial summation in a variety of sense modalities (Piéron, 1952).

It is important to note that the critical duration and trading function determined here apply to absolute threshold and not necessarily to supraliminal levels of constant warmth. It is always precarious to assume that measurements of threshold will predict the response of the sense organ to higher levels of stimulation. For example, the properties of spatial summation have been found to vary systematically from threshold warmth to levels that cause pain (Stevens \& Marks, 1971). Work in progress, using the method of magnitude estimation, suggests that the slope of the trading function decreases systematically with level of warmth, while the critical duration gets longer.

\section{Does Areal Extent Matter?}

Figure 3 compares the two trading functions obtained 
for comparatively large $\left(21.8 \mathrm{~cm}^{2}\right)$ and small $\left(5.72 \mathrm{~cm}^{2}\right)$ areas of the forehead. Except for position (i.e., absolute size of the threshold), the two functions look very similar in slope (about - 0.7 ) and critical duration (about $1.2 \mathrm{sec}$ ). The essential features of temporal summation do not, therefore, depend critically on area.

That the function for the smaller area lies higher on the graph reflects an important property of the warmth sense, namely, good spatial summation. The summation is nearly complete; i.e., the thresholds for an area $5.72 \mathrm{~cm}^{2}$ are consistently about three times larger than those for an area $\left(21.8 \mathrm{~cm}^{2}\right)$ that is about 3.8 times larger. This finding is in first-order agreement with various investigations of spatial summation (Hardy \& Oppel, 1937; Kenshalo, Decker, \& Hamilton, 1967; Stevens \& Marks, 1971). It also accords with the observation of Marks and Stevens (in press) that the rules governing spatial summation do not depend strongly on the duration of the stimulation. The relative independence of spatial summation and temporal summation make the life of the thermal psychophysicist a little simpler.

\section{Individual Differences}

Figure 4 presents the results of three Ss chosen to show how large the differences in apparent sensitivity can be. The main differences relate to the positions of the functions in $\log -\log$ coordinates, rather than their form or slope. In other words, these Ss differed greatly in absolute terms, but with respect to the rules by which time and intensity trade to preserve threshold, they behaved much alike. For example, no gross differences showed up in the critical duration. The data do not, however, rule out some variation. In fact, extensive pilot work on one $S$, who later became unavailable, suggested a critical duration of about $2 \mathrm{sec}$. Similarly, small variations in the slope of the trading function cannot be

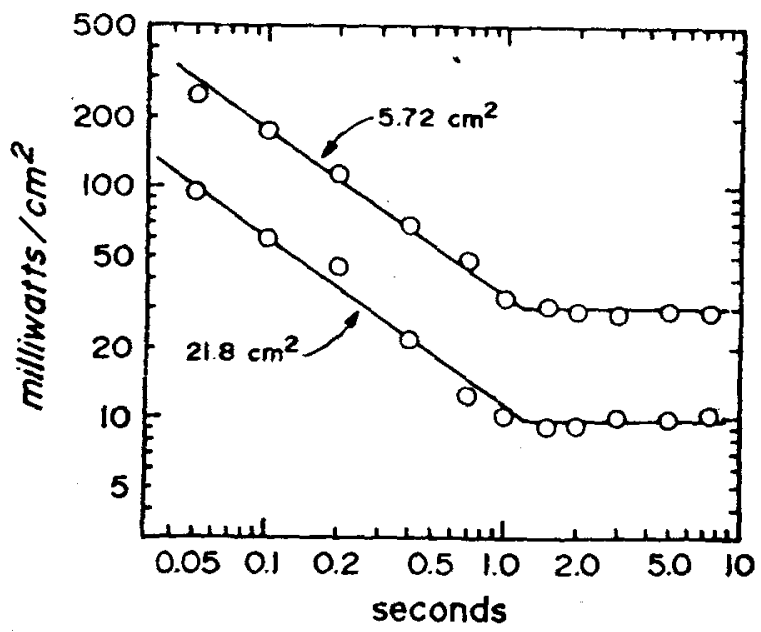

Fig. 3. Temporal summation at the warmth threshold for large $\left(21.8 \mathrm{~cm}^{2}\right)$ and small $\left(5.72 \mathrm{~cm}^{2}\right)$ areas. Plotted are the geometric averages for five sessions on one $S$.

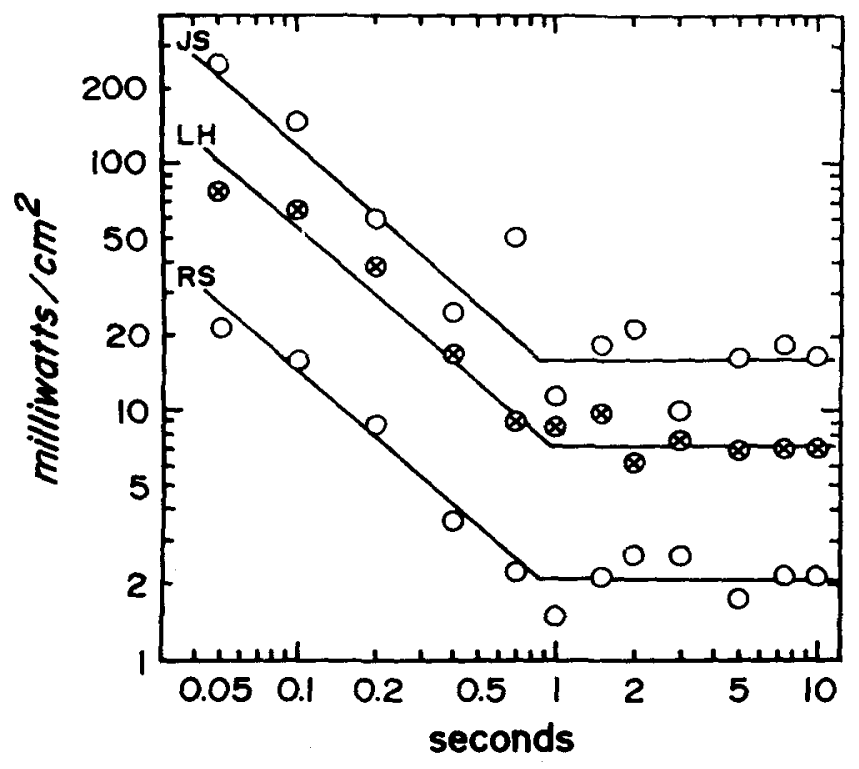

Fig. 4. Temporal summation at the warmth threshold for three individual $\mathrm{Ss}\left(21.8 \mathrm{~cm}^{2}\right.$ area $)$.

ruled out without more extensive testing. In any case, it is probably wise to examine more than one or two Ss if the goal is to learn how summation behaves in the average person.

Because of the large individual differences in absolute terms, a simple statistical measure of scatter is not very informative of "experimental error." For this reason, the data were first normalized and then analyzed for scatter, using the following procedure. First, all threshold levels were converted to logarithms. Then, for each S's data, a constant was added to each value. This constant was chosen such that it made a given S's value for the 5-sec duration equal to the mean for the group. Finally, the standard deviations of the log thresholds were computed and their antilogarithms plotted in Fig. 2 (vertical lines represent plus and minus one standard deviation).

Individual differences may help to resolve some of the discrepancies among studies of temporal summation. The position in Fig. 1 of the function determined by Geblewicz (1935) may be too high, however, to be reasonably explained in that way. Moreover, the "square root" character of the function (dotted line) rests heavily on the point at $4 \mathrm{sec}$-which seems in the light of all the other studies to lie beyond critical duration. The solid line (slope $=-0.7$ ) drawn by the present writers would seem to offer a reasonable alternative description of Geblewicz's data.

The data of Hendler, Hardy, and Murgatroyd (1963) yield a slope of approximately -1.1 , thereby suggesting a kind of "supersummation." Occasionally we obtained data that could be approximated by slopes in the neighborhood of -1.0 , but a slope slightly smaller than that was the typical result.

There is good a priori reason to suspect that the rules by which irradiance and duration trade might depend on 


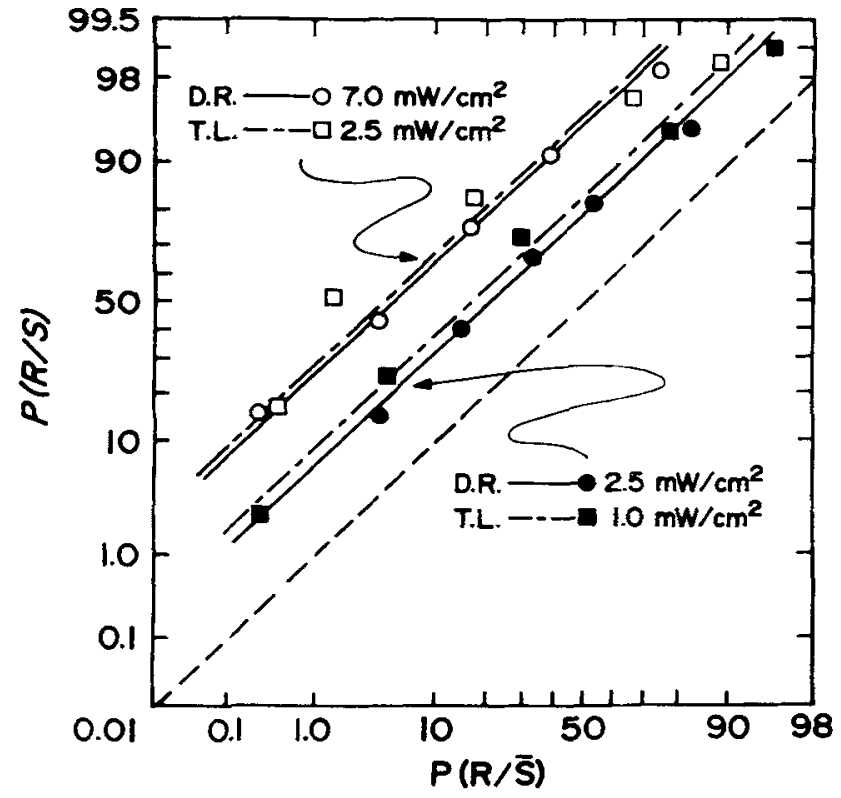

Fig. 5. ROCs for the detectability of thermal irradiation by two individual Ss. S T.L. is more than twice as sensitive as D.R.

the spectrum of the irradiation. Microwaves penetrate into deep tissues, whereas infrared waves tend to get absorbed near the skin surface (in the case of skin blackened with India ink, all radiation is absorbed near the surface). It is therefore surprising and perhaps significant that the slope in Fig. 1 of the trading function determined by Eijkman and Vendrik (1961) is nearly the same $(-0.7)$ as that determined in the present study. The probable significance of this finding is discussed below in terms of the thermal events that take place in the skin when it is heated by irradiation.

In conclusion, seen in the light of results from six Ss, the three functions in Fig. 1 obtained from three different laboratories do not differ so much in their essential features that they cannot reasonably be put down to individual differences among Ss.

\section{Signal Detection}

Figure 5 shows ROCs based on the confidence rating of two Ss, D.R. and T.L., who were selected for this task because they appeared to differ so much in sensitivity when tested by the method of limits. The points were determined by the procedure described by Green and Swets (1966). This procedure treats each boundary between response categories as a given criterion level. Except for T.L.'s data at $2.5 \mathrm{~mW} / \mathrm{cm}^{2}$ (unfilled squares), the points could be well fitted by lines of unit slope in double probability coordinates. Such slopes are consistent with the notion that the underlying distributions are normal and of equal variance.

Although the unfilled squares furnish a more complicated case, it is clear that they lie in close proximity to D.R.'s data for $7.0 \mathrm{~mW} / \mathrm{cm}^{2}$ (unfilled circles). The lower pair of functions (filled symbols) contribute nearly the same information without ambiguity: D.R.'s detection of $2.5 \mathrm{~mW} / \mathrm{cm}^{2}$ was slightly poorer than T.L.'s detection of $1.0 \mathrm{~mW} / \mathrm{cm}^{2}$. T.L. seems, therefore, to be about 2.5 times as sensitive as D.R.

The results argue that there is a real and fairly large difference between the two Ss. Not nearly so large, though, as the tenfold difference given by the method of limits with criterion uncontrolled. In any case, as demonstrated by Fig. 4, the absolute differences among Ss, whether due to criterial or to real sensory causes, need not obscure the nature of temporal summation, because it is the relative sensitivity that really matters.

\section{Relation to Changes in Skin Temperature}

It is possible to take a more proximal view of the stimulus, i.e., to view the stimulus as a change in skin temperature instead of a level of irradiance. To that end, changes in skin temperature were calculated with the use of a model (for complete details see Stolwijk \& Hardy, 1965; Marks \& Stevens, 1968) that simulates the thermal state of the skin. Stolwijk and Hardy's model divides the skin into eight layers from the surface to a depth of $1.1 \mathrm{~cm}$ and gives eight linear thermodynamic equations, one for each layer. The constants of the equations were determined from measured thermal properties of the skin. Each equation contains one term for heat flow to the layer above it, another for heat flow to the layer below. The deepest layer is assumed to absorb constant metabolic heat, and the most superficial layer is assumed to absorb all of the incident radiation. (Because the skin was blackened, all of the radiation was absorbed near the skin's surface). Changes in skin temperature were calculated on a digital computer by means of the method of finite differences.

The circles in Fig. 6 show the increases in temperature $(\Delta T)$ at the skin surface produced by threshold levels of irradiance, plotted as functions of stimulus duration. Since threshold irradiance was constant over long durations, it is not surprising that $\Delta T$ increased when duration exceeded $1 \mathrm{sec}$, since, as was mentioned above, with constant irradiance skin temperature rises continuously. In fact, the size of $\Delta \mathrm{T}$ was smallest between about 0.5 and $1 \mathrm{sec}$, greater both at shorter and at longer durations. The fact that $\Delta \mathrm{T}$ at the skin surface was not constant is not disappointing, since it is unlikely that warmth receptors are located right at the surface. Figure 6 also gives $\Delta T$ calculated at two depths below the surface: $0.175 \mathrm{~mm}$ (squares) and $0.275 \mathrm{~mm}$ (circles).

According to the Stolwijk-Hardy model, when the skin is irradiated, surface temperature rises continuously until the stimulus is terminated, after which time surface temperature declines; but, in contrast, subcutaneous temperatures continue to rise over short periods of time after termination of the stimulus. This additional 
increase can be substantial: given a $50-\mathrm{msec}$ pulse, for example, temperature at a depth of $0.175 \mathrm{~mm}$ increases over a total period of $150 \mathrm{msec}$, at which point $\Delta \mathrm{T}$ is 10 times as great as it was when the stimulus terminated.

The unfilled symbols in Fig. 6 show $\Delta T$ at the point in time when the stimulus terminated. The filled symbols show the maximal $\Delta \mathrm{T}$, i.e., the $\Delta \mathrm{T}$ reached at some point later in time. Clearly, the relative difference between open and filled symbols is greatest at short durations and decreases as duration increases. The maximal values of $\Delta \mathrm{T}$ appear the more appropriate for consideration. At a depth of $0.175 \mathrm{~mm}, \Delta \mathrm{T}$ (filled squares) was essentially constant over the first $0.5 \mathrm{sec}$, after which $\Delta T$ rose. This outcome suggests that warmth receptors may be located at that depth and that threshold warmth corresponds to an increase in tissue temperature of about $0.015^{\circ} \mathrm{C}$. A similar conclusion was reached by Hendler, Hardy, and Murgatroyd (1963) on the basis of thresholds measured for infrared irradiation. They placed receptors at a depth of about $0.2 \mathrm{~mm}$.

When warmth thresholds are measured with microwave irradiation, however, $\Delta \mathrm{T}$ is not constant at any depth (Eijkman \& Vendrik, 1961; Hendler, Hardy, \& Murgatroyd, 1963). Hendler et al were led to reject constant $\Delta \mathrm{T}$ as the critical stimulus; instead, they suggested that the stimulus for warmth may be the difference between temperatures at two points in the skin $(0.2$ and $1.0 \mathrm{~mm})$. The present results are consistent with that "two-layer" hypothesis: since the temperature at a depth of $1 \mathrm{~mm}$ hardly changes under infrared irradiation unless it is intense or prolonged, the two-layer hypothesis applies as well as does a single-layer model.

An alternative to the two-layer hypothesis is that of Eijkman and Vendrik (1961), who suggested that adaptation serves more and more to offset the effect of rising skin temperature as duration increases. Such a process could explain the increase in $\Delta T$ that takes place as duration increases beyond $0.5 \mathrm{sec}$. Eijkman and Vendrik's data required posţulation of a second adaptational process, one that acts primarily over the shorter durations. The assumption that there are two adaptational processes is consistent with the present results if warmth receptors are assumed to lie at a depth greater than $0.175 \mathrm{~mm}$. At a depth of $0.275 \mathrm{~mm}$, for example, the relation between $\Delta \mathrm{T}$ and duration shown in Fig. 6 is similar to the one implied by Eijkman and Vendrik's data obtained with microwave stimulation.

\section{CONCLUSION}

It would seem, then, that three psychophysiological hypotheses can explain the present data on summation: (1) Threshold is reached when the radiant stimulus produces a fixed increase in skin temperature (depth of about $0.2 \mathrm{~mm}$ ); (2) threshold is reached when the stimulus produces a fixed increase in the difference between two skin temperatures (depths of about 0.2 and

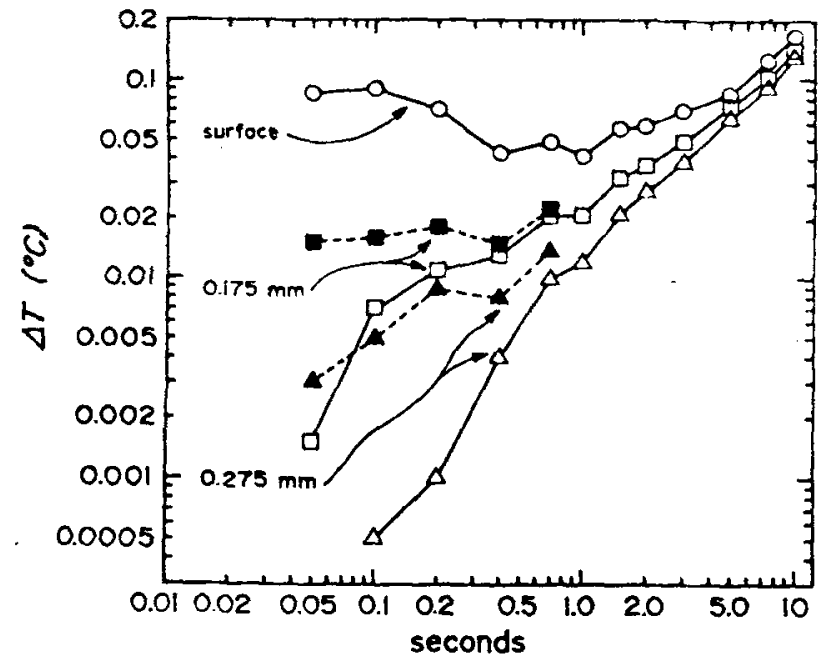

Fig. 6. Increases in skin temperature $(\Delta T)$ brought about by threshold levels of imadiance, as calculated from the model of Stolwijk and Hardy (1965). Circles: $\Delta T$ at the skin surface. Squares: $\Delta T$ at a depth of $0.175 \mathrm{~mm}$. Triangles: $\Delta T$ at a depth of $0.275 \mathrm{~mm}$. Unfilled symbols give $\Delta T$ at the end of the stimulus exposure; filled symbols give the maximal $\Delta T$ reached.

$1.0 \mathrm{~mm}$ ); (3) threshold is reached when the stimulus produces an increase in skin temperature (depth greater than about $0.2 \mathrm{~mm}$ ) whose size depends on the extent of concurrent adaptation. Thresholds for microwaves (Eijkman \& Vendrik, 1961; Hendler, Hardy, \& Murgatroyd, 1963) seem compatible with only (2) and (3).

Regardless of which, if any, of these three hypotheses turns out to be correct, the psychophysics of warmth summation resembles that of the other senses. Thresholds for vision (Bartlett, 1965), hearing (Garner, 1947), taste (Bujas, 1935), vibration (Verrillo, 1965), rotary acceleration (Clark \& Stewart, 1968), and cold (Bujas, 1938) all depend on duration over some limited range, so that there is an inverse relation between intensity and duration. In vision, the reciprocity is perfect, i.e., the eye sums energy (Bloch's law). For most sense modalities, however, summation seems to be not quite perfect. To offset a given percentage change in intensity requires a slightly larger percentage change in duration.

Beyond a critical duration, duration no longer matters, and threshold becomes constant (critical duration ranges all the way from about $0.1 \mathrm{sec}$ for vision to about $3 \mathrm{sec}$ for taste and maybe even longer for rotary acceleration). Why does summation cease at a critical duration? In a way, it is easier to explain summation than to explain its failure beyond the critical duration. All the senses pose this same problem. Perhaps it will be necessary for any hypothesis about summation to invoke, as do Eijkman and Vendrik, the concept of sensory adaptation, such that any change brought about too slowly will be rendered ineffective by a decline in sensitivity over time. 


\section{REFERENCES}

Bartlett, N. R. Thresholds as dependent on some energy relations and characteristics of the subject. In C. H. Graham (Ed.), Vision and visual perception. New York: Wiley, 1965. Pp. 154-184.

Bujas, Z. Le rapport entre les quantités liminaires et le temps d'action pour les excitations gustatives. Comptes Rendus des Sciences de la Societé de Biologie, 1935, 119, 835-837.

Bujas, Z. La sensibilité au froid en fonction du temps. L'Année Psychologique, 1938, 38, 140-147.

Clark, B., \& Stewart, J. D. Magnitude estimates of rotational velocity during and following prolonged increasing, constant, and zero angular acceleration. Journal of Experimental Psychology, 1968, 78, 329-339.

Eijkman, E., \& Vendrik, A. J. H. Dynamic behavior of the warmth sense organ. Journal of Experimental Psychology, $1961,62,403-408$.

Garner, W. R. The effect of frequency spectrum on temporal integration of energy in the ear. Journal of the Acoustical Society of America, 1947, 19, 808-815.

Geblewicz, E. La relation du temps d'action liminaire avec l'intensité pour les stimulations thermiques. Comptes Rendus des Sciences de la Societé de Biologie, 1935, 118, 748-750.

Green, D. M., \& Swets, J. Signal detection theory and psychophysics. New York: Wiley, 1966.

Gundy, R. F. Auditory detection of an unspecified signal. Journal of the Acoustical Society of America, 1961, 33, 1009-1012.

Hardy, J. D., \& Oppel, T. W. Studies on temperature sensation. III. The sensitivity of the body to heat and the spatial summation of the end organ responses. Journal of Clinical Investigation, $1937,16,533-540$.

Hardy, J. D., Wolff, H. G., \& Goodell, H. Pain sensations and reactions. Baltimore: Williams \& Wilkins, 1952.

Hendler, E., Hardy, J. D., \& Murgatroyd, D. Skin heating and temperature sensation produced by infrared and microwave irradiation. In C. M. Herzfeld (Ed.), Temperature: Its measurement and control in science and industry. Vol. 3, Part 3. New York: Reinhold, 1963. Pp. 211-230.

Kenshalo, D. R., Decker, T., \& Hamilton, A. Spatial summation on the forehead, forearm, and back produced by radiant and conducted heat. Journal of Comparative \& Physiological Psychology, 1967, 63, 510-515.

Marks, L. E., \& Stevens, J. C. Perceived warmth and skin temperature as functions of the duration and level of thermal irradiation. Perception \& Psychophysics, 1968, 4, 220-228.

Marks, L. E., \& Stevens, J. C. Spatial summation of warmth: Influence of duration and configuration of the stimulus. American Journal of Psychology, in press.

Piéron, $\mathrm{H}$. The sensations. New Haven: Yale University Press, 1952.

Stevens, J. C., \& Marks, L. E. Spatial summation and the dynamics of warmth sensation. Perception \& Psychophysics, 1971, 9, 391-398.

Stolwijk, J. A. J., \& Hardy, J. D. Skin and subcutaneous temperature changes during exposure to intense thermal radiation. Journal of Applied Physiology, 1965, 20, 1006-1013.

Verrillo, R. T. Temporal summation in vibrotactile sensitivity, Journal of the Acoustical Society of America, 1965, 37, 843-846.

\section{NOTE}

1. One additional study (Hendler, Hardy, \& Murgatroyd, 1963) varied the duration of microwave stimulation in the determination of warmth thresholds. The data are omitted from Fig. 1 because only a couple of durations were short enough to fall unequivocally in the region of temporal summation.

(Received for publication February 20, 1973; accepted March 3, 1973.) 